\title{
Development of the LBNL Positron Emission Mammography Camera
}

\author{
J.S. Huber, Member, IEEE, W.S. Choong, Member, IEEE, J. Wang, Member, IEEE, J.S. Maltz, \\ Member, IEEE, J. Qi, Member, IEEE, E. Mandelli, Member, IEEE, and W.W. Moses, Senior \\ Member, IEEE.
}

\begin{abstract}
We present the construction status of the LBNL Positron Emission Mammography (PEM) camera, which utilizes a PET detector module with depth of interaction measurement consisting of 64 LSO crystals $\left(3 \times 3 \times 30 \mathrm{~mm}^{3}\right)$ coupled on one end to a single photomultiplier tube (PMT) and on the opposite end to a 64 pixel array of silicon photodiodes (PDs). The PMT provides an accurate timing pulse, the PDs identify the crystal of interaction, the sum provides a total energy signal, and the $\mathrm{PD} /(\mathrm{PD}+\mathrm{PMT})$ ratio determines the depth of interaction. We have completed construction of all 42 PEM detector modules. All data acquisition electronics have been completed, fully tested and loaded onto the gantry. We have demonstrated that all functions of the custom IC work using the production rigid-flex boards and data acquisition system. Preliminary detector module characterization and coincidence data have been taken using the production system, including initial images.
\end{abstract}

\section{INTRODUCTION}

$\mathrm{T}$ his paper describes the status and construction of the LBNL Positron Emission Mammography (PEM) camera [1]. The geometry for the PEM camera is shown in Fig. 1. The breast is inserted in the gap between the top and bottom $7.5 \times 10 \mathrm{~cm}^{2}$ detector planes, and the spacing between planes adjusted to provide light compression on the breast. The positions of the $7.5 \times 7.5 \mathrm{~cm}^{2}$ detector planes on the left and right of the breast are fixed. The volumes represent the active portion of the detector (i.e., the portion sensitive to $511 \mathrm{keV}$ photons), which are divided into $2.5 \times 2.5 \times 3.0 \mathrm{~cm}^{3}$ modules. Each module is further divided into 64 individual LSO scintillation crystals, each $3 \times 3 \times 30 \mathrm{~mm}^{3}$. We have currently instrumented only the two side $\left(7.5 \times 7.5 \mathrm{~cm}^{2}\right)$ detector planes, using 18 detector modules, which are shown in Fig. 1 as dark gray.

We have previously reported on a PET detector module capable of measuring depth of interaction on an event by event basis [2, 3], shown in Fig. 2. With this design, a single photomultiplier tube (PMT) provides an accurate timing pulse and initial energy discrimination for an $8 \times 8$ array of LSO [4] scintillator crystals, an $8 \times 18$ silicon

Manuscript received December, 2002. This work was supported in part by the U.S. Department of Energy under contract No. DE-AC0376SF00098, in part by Public Health Service Grant Nos. P01-HL25840, RO1-CA67911, and RO1-NS29655, and in part by Breast Cancer Research Program of the University of California Grant no 1RB-0068.

J. S. Huber, W. S. Choong, J. Wang, J. S. Maltz, J. Qi, E. Mandelli, and W. W. are with the Lawrence Berkeley National Laboratory, Mailstop 55121, 1 Cyclotron Road, Berkeley, CA 94720 USA (telephone: 510-4866445, e-mail: jshuber@1bl.gov). photodiode array (PDs) identifies the crystal of interaction, the $\mathrm{PD} /(\mathrm{PD}+\mathrm{PMT})$ ratio measures the depth of interaction, and the sum (PD+PMT) provides a total energy signal. We are planning to use this PET detector module in several tomograph designs $[1,5,6]$; thus we have transformed our prototype detector into one that can be produced in quantity.

We focus in this paper on new results using production versions of the complete system -- custom ICs, rigid-flex boards (used to read out the IC), detector modules, and data acquisition system. The major milestone of reading out coincidence data with the production system has been achieved, and initial images are shown.

\section{SYSTEM}

\section{A. Detector Modules}

We have built all 42 production detector modules to be used in our PEM camera design [1]. Fig. 2 shows a photograph of one production detector module. The end-on "profile" of the module is $2.5 \mathrm{~cm}$ square, allowing close packing of multiple detector modules in two dimensions. Communication between the custom integrated circuit (PETRIC) [7] and the remainder of the electronics [8] (which is located "behind" the photomultiplier tube) is accomplished by a 0.008 " thick Kapton "flex tail" on the IC printed circuit board that is thin enough to run between adjacent detector modules. The successful production of these rigid-flex boards has proved to be a surprisingly difficult task, but we are now taking data using the working, final versions of the rigid-flex boards. All functions of the PETRIC chip (including the Winner-Take-All circuitry for correct analog and channel address selection of the "winner") have been demonstrated to work with production rigid-flex boards and the final data acquisition system.

Fig. 3 demonstrates that the detector module assembly process is complete. We are now in the process of attaching rigid-flex boards onto each detector module's photodiode, as well as bundling three detector modules (with rigid-flex boards) and assembling them into light tight cans. Fig. 4 shows the details of our "can" assembly. Currently, half of the detector modules have been assembled into cans.

\section{B. Camera}

We have instrumented the two side $\left(7.5 \times 7.5 \mathrm{~cm}^{2}\right)$ detector planes, using 18 detector modules (i.e., 3 detector 
cans per bank). Fig. 5 shows the camera gantry and electronics housing before the modules were put in place.

The detector modules are read out with electronics originally developed by CTI (Knoxville, TN) for the HRRT brain imaging PET camera and modified to use a detector module that incorporates a photodiode [8]. The top level diagram of the electronics system is show in Fig. 6a, and the diagram of the Detector Head Interface (DHI) board is shown in Fig. 6b. The outputs of three detector modules are processed by an Analog Subsection board. Events are detected and assigned an arrival time, the crystal of interaction is identified, energy qualification is performed, and a digital word is formed. The output signals from the Analog Subsection boards are then multiplexed by Detector Head Interface boards. A CTI Coincidence Processor identifies singles events in the different DHI boards that are in coincidence, and the output is sent to a Pentium-based computer system that accumulates the coincident data, reconstructs the images, and displays them.

This final data acquisition system was used to calibrate the detector modules. We have automated calibration software that sets up the electronics and identifies bad channels using test pulses. In addition, we have automated software that acquires (singles or coincidence) data with a ${ }^{68} \mathrm{Ge}$ source (using the PMT signal with a low discriminator threshold for triggering), corrects for channel to channel gain variation in the PMT signal, and cuts events with PMT signal outside the 350-700 keV energy window. However, we currently have not corrected for channel to channel gain variation in the PD signal, and have not scaled the relative PD and PMT signal; thus we have not yet been able to calculate the depth of interaction (DOI) with the production system. Since the calibration procedure is not complete [9], the PD is only used to identify the crystal of interaction.

\section{Reconstruction}

We have developed and tested both filtered backprojection reconstruction algorithms (2-D [10] and 3-D [11]) and a list mode regularized maximum likelihood reconstruction algorithm [12] using Monte Carlo data. The rectangular geometry and the capability of measuring DOI result in irregular sampling in the sinogram space. In the filtered backprojection algorithm, data are rebinned onto regular grid. In the list mode maximum likelihood algorithm, the irregular sampling and DOI information are explicitly modeled in the projection matrix. To demonstrate the expected system performance, Fig. 7 shows a reconstructed image of simulated spherical sources using the $3 \mathrm{D}$ filtered backprojection algorithm. The resolution of the reconstruction varies from $1.54 \mathrm{~mm}$ FWHM at the center to $3.10 \mathrm{~mm}$ at the corner. As the current camera setup only contains two opposing detector banks, the filtered backprojection algorithm is not directly applicable. Therefore, in Section III we only show the list mode maximum likelihood reconstruction of the real data.

\section{RESULTS}

The two instrumented detector banks were used to image ${ }^{68} \mathrm{Ge}$ sources in two different configurations, as shown in Fig. 8. The distance between the opposing detector banks was 7 $\mathrm{cm}$. In the first setup, a $1.5 \mathrm{~mm}$ diameter ${ }^{68} \mathrm{Ge}$ point source $(106 \mu \mathrm{Ci})$ was placed in the mid-plane between the two planar detector banks. Data was taken at two different point source positions (Fig. 8a), resulting in 10M events each. The images, shown in Fig. 9, were reconstructed with the maximum likelihood algorithm using $1.5 \times 1.5 \times 3 \mathrm{~mm}^{3}$ voxels. Normalization factors were measured using a plane source placed in the mid-plane. The reconstructed point in position 1, as shown in Fig. 9a, has a $(1.65,2.85) \mathrm{mm}$ FWHM reconstruction resolution. In position 2, the reconstruction resolution is $(2.40,1.95) \mathrm{mm}$ FWHM. The variation in FWHM is mostly caused by the misalignment between the point source position and the voxel grid. The non-negativity constraint in the maximum likelihood reconstruction did not have a big impact on the resolution measurement here, as we obtained similar results when we reconstructed the point source data after superimposing it with a simulated uniform background.

In the second setup, a point and rod source in different planes were used. The $1.5 \mathrm{~mm}$ diameter ${ }^{68} \mathrm{Ge}$ point source $(106 \mu \mathrm{Ci})$ was placed in the mid-plane and a $2.5 \mathrm{~mm}$ diameter rod source $(118 \mu \mathrm{Ci})$ was placed in a plane that was $2 \mathrm{~cm}$ apart (Fig. 8b). Fig. 10 shows the images of the rod and point source with $10 \mathrm{M}$ events. The point source has $(3.00,2.25) \mathrm{mm}$ FWHM reconstruction resolution. Since the concentration of activity in the point source is much stronger than the rod source, background activity in the area of the point source is seen in the rod source plane.

Fig. 11 shows the spectra of the timing difference between two production detector modules, which were read out with the final data acquisition system. Data was taken using the $1.5 \mathrm{~mm}$ diameter ${ }^{68} \mathrm{Ge}$ point source $(106 \mu \mathrm{Ci})$ placed in the mid-plane between the two detector modules. Only the PMT information was used, with a low energy threshold. While channel to channel corrections are planned, none were used to acquire these data. The preliminary coincidence timing resolution of $4.8 \mathrm{nsec}$ is expected to improve after timing calibration is complete.

\section{DISCUSSION AND CONCLUSION}

Two significant tasks remain before this camera is complete: assembly of the remaining two detector banks needs to be finished and the remainder of the calibration algorithms (especially PD) needs to be developed. However, we feel that major milestones have been achieved with our system. All components are complete -- custom ICs, flex boards, detector modules, data acquisition electronics and the gantry. Production firmware and calibration has been developed. Most importantly, the first images have been acquired using two opposing banks of $3 \times 3$ modules each. Initial reconstruction resolutions are in agreement with expectation.

\section{ACKNOWLEDGMENT}

This work was supported in part by U.S. Department of Energy under contract No. DE-AC03-76SF00098, and in part 
by the Public Health Service Grant Nos. P01-HL25840 and R01-CA67911. Reference to a company or product name does not imply approval or recommendation by the University of California or the U.S. Department of Energy to the exclusion of others that may be suitable.

\section{REFERENCES}

1. W. W. Moses, T. F. Budinger, R. H. Huesman, et al., "PET camera designs for imaging breast cancer and axillary node involvement," $J$ Nucl Med, vol. 36, pp. 69P (abstract), 1995.

2. J. S. Huber, W. W. Moses, S. E. Derenzo, et al., "Characterization of a 64 channel PET detector using photodiodes for crystal identification," IEEE Trans Nucl Sci, vol. NS-44, pp. 1197-1201, 1997.

3. J. S. Huber, W. W. Moses, M. S. Andreaco, et al., "A LSO Scintillator Array for a PET Detector Module with Depth of Interaction Measurement," IEEE Trans Nucl Sci, vol. NS-48, pp. 684-688, 2001.

4. C. L. Melcher and J. S. Schweitzer, "Cerium-doped lutetium oxyorthosilicate: a fast, efficient new scintillator," IEEE Trans Nuc Sci, vol. 39, pp. 502-5, 1992.

5. W. W. Moses, P. R. G. Virador, S. E. Derenzo, et al., "Design of a high-resolution, high-sensitivity PET camera for human brains and small animals," IEEE Trans. Nucl. Sci., vol. NS-44, pp. 1487-1491, 1997.

6. J. S. Huber and W. W. Moses, "Conceptual design of a high sensitivity small animal PET camera with $4 \pi$ coverage," IEEE Trans Nucl Sci, vol. NS-46, pp. 498-502, 1999.

7. M. Pedrali-Noy, G. J. Gruber, B. Krieger, et al., "PETRIC - A Positron Emission Tomography Readout IC," IEEE Trans Nucl Sci, vol. NS-48, pp. 860-864, 2000.

8. W. W. Moses, J. W. Young, K. Baker, et al., "The Electronics System for the LBNL Positron Emission Mammography (PEM) Camera," IEEE Trans Nucl Sci, vol. NS-48, pp. 632-636, 2001.

9. J. S. Huber, W. W. Moses, and P. R. G. Virador, "Calibration of a PET detector module that measures depth of interaction," IEEE Trans Nucl Sci, vol. NS-45, pp. 1268-72, 1998.

10. P. R. G. Virador, W. W. Moses, and R. H. Huesman, "Reconstruction in PET cameras with irregular sampling and depth of interaction capability," IEEE Trans Nucl Sci, vol. NS-45, pp. 1225-30, 1998.

11. P. R. G. Virador, W. W. Moses, R. H. Huesman, et al., "3D Reconstruction in PET Cameras with Irregular Sampling and Depth of Interaction," IEEE Trans. Nucl. Sci., vol. NS-48, pp. 1524-1529, 2001.

12. R. H. Huesman, G. J. Klein, W. W. Moses, et al., "List mode maximum likelihood reconstruction applied to positron emission mammography with irregular sampling.," IEEE Trans Med Imag, vol. 19 , pp. 532-537, 2000 


\section{Figures:}

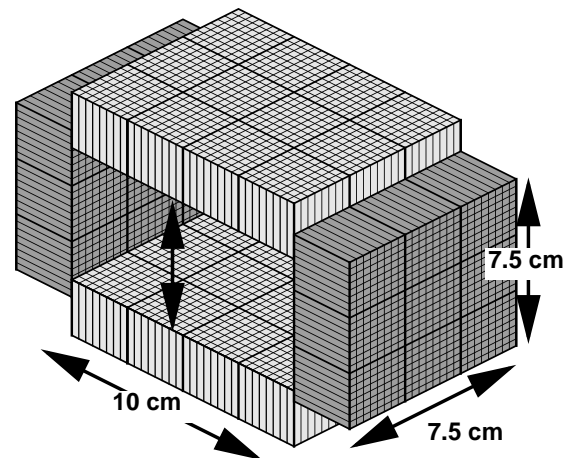

Fig. 1. Positron Emission Mammography (PEM) camera [1]. The breast is inserted in the gap between the top and bottom $7.5 \times 10 \mathrm{~cm}^{2}$ detector planes, and the spacing between planes adjusted to provide light compression on the breast. The positions of the $7.5 \times 7.5 \mathrm{~cm}^{2}$ detector planes on the left and right of the breast are fixed. The volumes represent the active portion of the detector (i.e., the portion sensitive to $511 \mathrm{keV}$ photons), which are divided into $2.5 \times 2.5 \times 3.0 \mathrm{~cm}^{3}$ modules. We have currently instrumented only the two side $(7.5 \mathrm{~cm} \times 7.5 \mathrm{~cm})$ detector planes, using 18 detector modules, which are shown in dark gray.

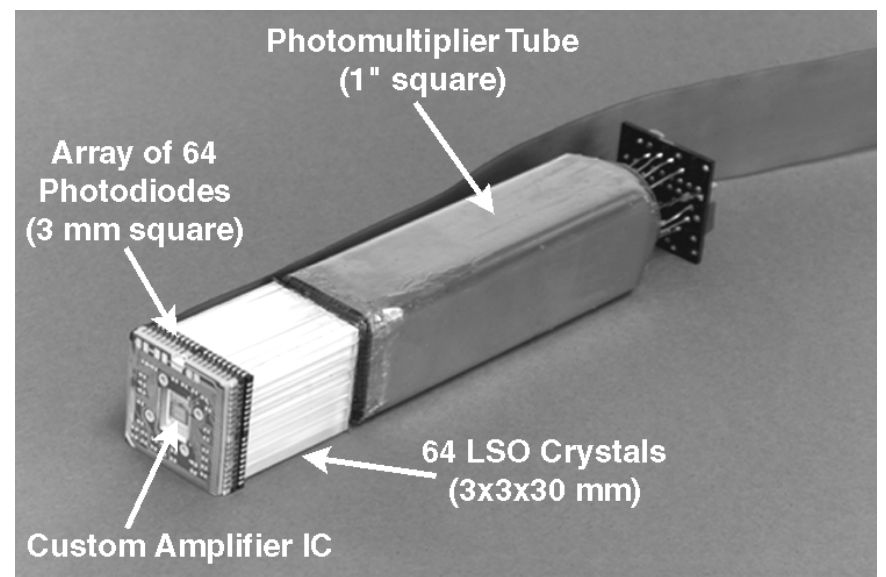

Fig. 2. Photograph of the PET detector. Each crystal is attached to a photomultiplier tube which provides timing and initial energy information, and to a photodiode which identifies the crystal of interaction. The PMT and PD signals are combined to measure the depth of interaction and total energy. The custom integrated circuit is mounted on a $2.5 \mathrm{~cm}$ square circuit board with a 0.008 " thick Kapton tail, which goes between adjacent detector modules and acts as a cable connecting the IC to the remainder of the readout electronics.

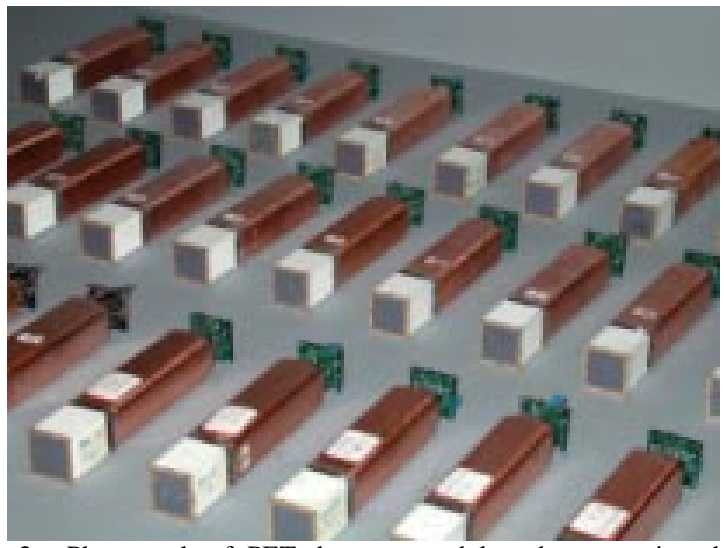

Fig. 3. Photograph of PET detector modules, demonstrating that the detector module assembly is complete. We have built all 42 detector modules that are needed for the PEM camera.

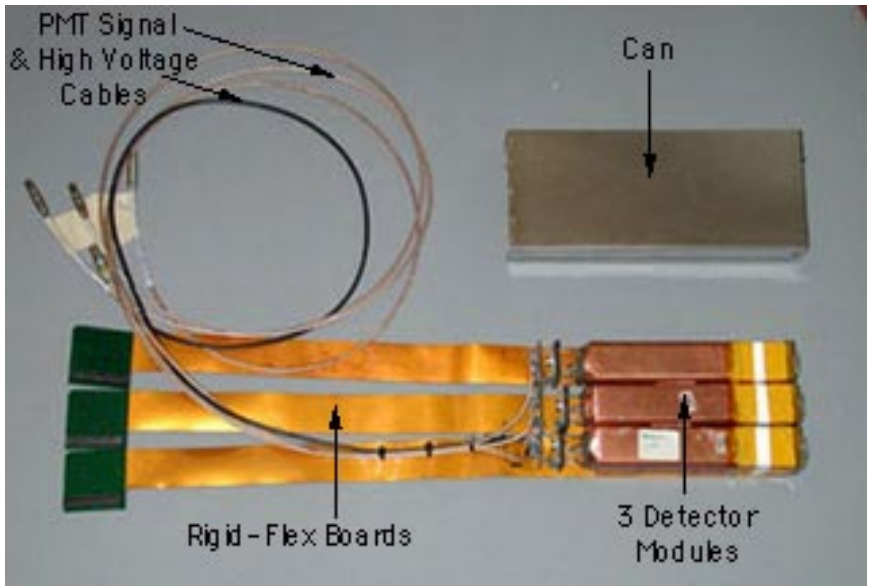

Fig. 4. Photograph of the detector "can" assembly. Rigid-flex boards are attached to each detector module's photodiode. Three detector modules are then attached to a high voltage distribution board and assembled into a light-tight can.

(a)

(b)
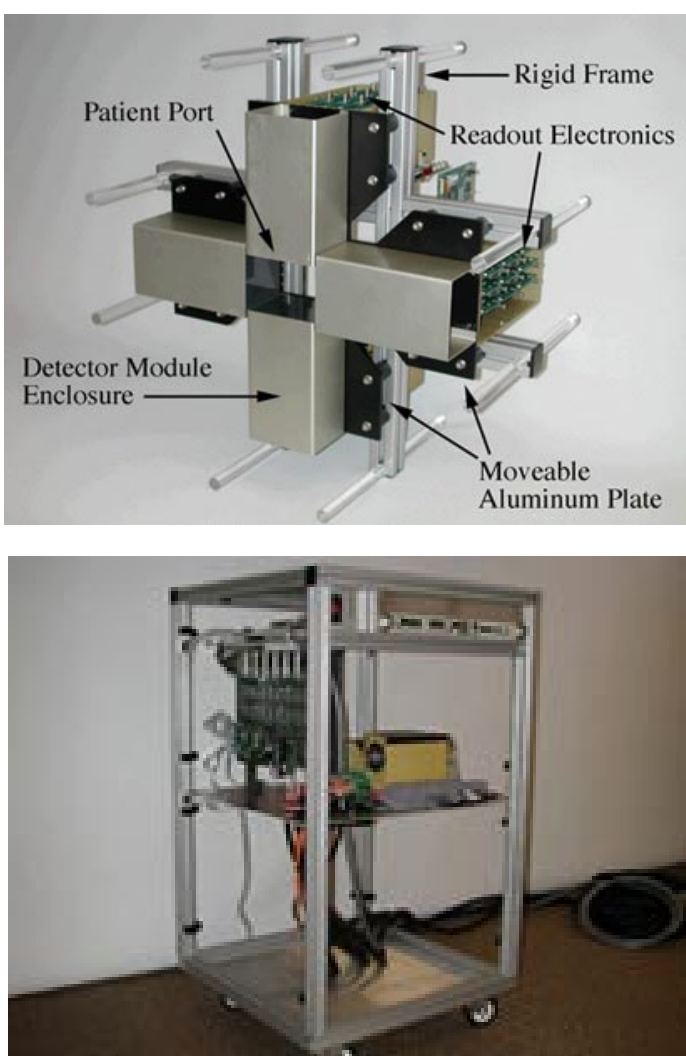

Fig. 5. (a) A photograph of the actual PEM gantry which is fully loaded with the complete data acquisition electronics (but not loaded with detector modules). Each detector bank utilizes nine (side bank) or twelve (top/bottom bank) detector modules. The detector modules are loaded into a can (Fig 4) with the Kapton "flex tail" feeding out between the detector modules and connecting to the readout electronics. (b) PEM camera electronics cabinet. There is space for the computer on the bottom. The Coincidence Processor and power supplies are visible in the middle area, and the HV power panel is seen on the top. 
(a)

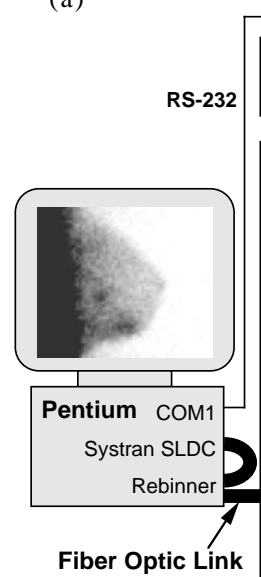

(b)

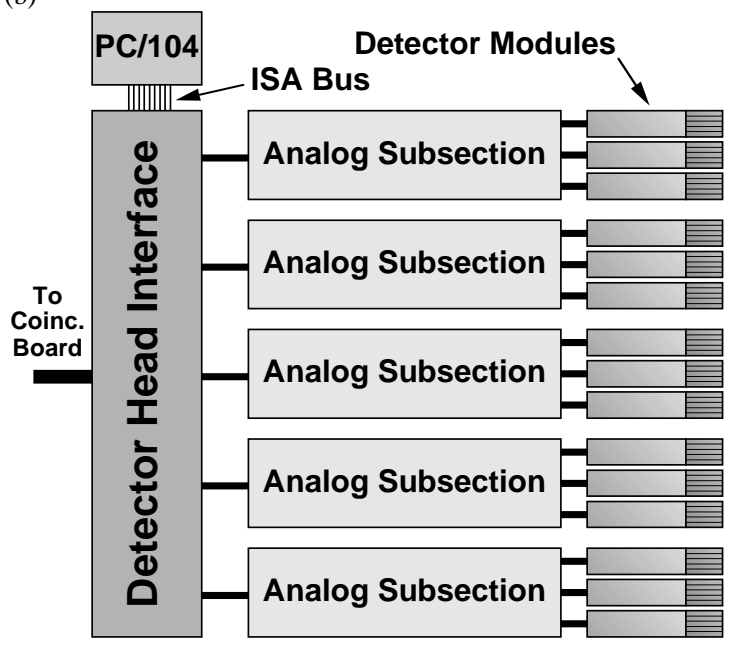

Fig. 6. (a) Top level diagram of the electronics system. Each of the four Detector Head Interface boards collects "singles" events which are forwarded to the Coincidence Processor. Coincident events are then passed to the Pentium-based data acquisition computer via a fiber optic link to a Systran SLDC receiver. A rebinner board can optionally be placed between the Coincidence Processor and the fiber optic readout board. (b) Diagram of the Detector Head Interface (DHI). Three detector modules are serviced by each Analog Subsection board. The singles event words generated by the Analog Subsection board are multiplexed by the DHI board, which also buffers communication with the PC/104 as well as system timing signals [8].

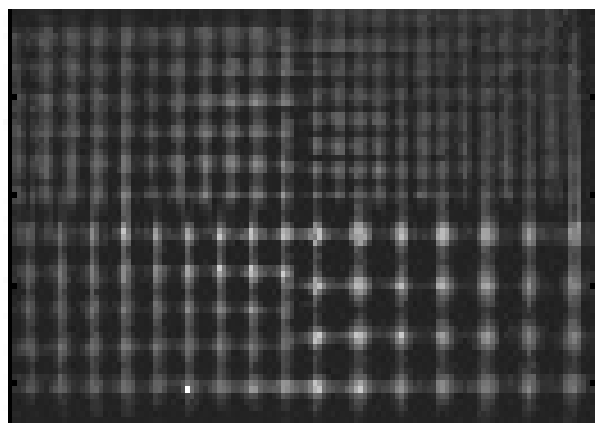

Fig. 7 Simulated sphere sources located at the central plane. The spheres are arranged where the distances between the sources are four times their diameters (like a Derenzo Phantom). The diameters are $1.0 \mathrm{~mm}$, $1.25 \mathrm{~mm}, 1.5 \mathrm{~mm}$, and $2.0 \mathrm{~mm}$. (a)

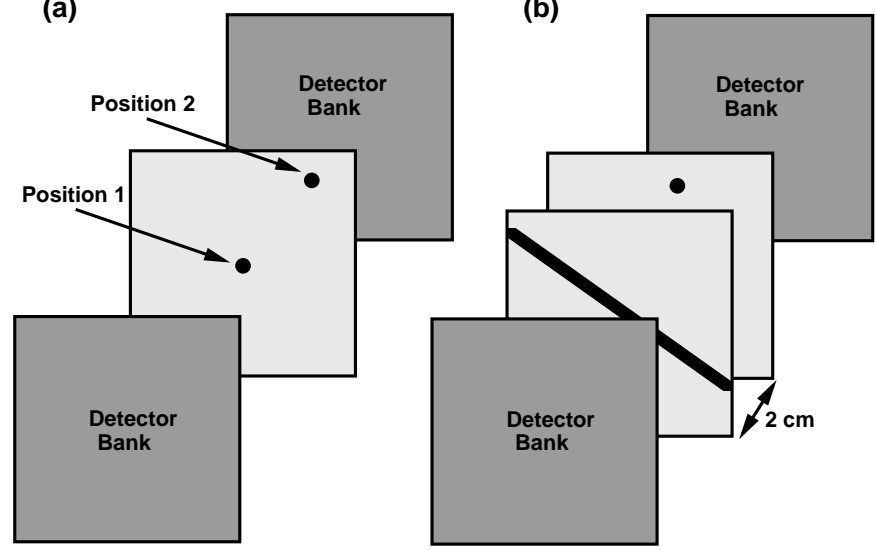

Fig. 8. Geometry setup for data taking, with ${ }^{68} \mathrm{Ge}$ sources placed between two opposing planar detector banks. The distance between detector banks was $7 \mathrm{~cm}$. (a) In the first setup, a point source in the midplane was used. Data was taken at two different point source positions. (b) In the second setup, a point and rod source in different planes were used. The point source $(1.5 \mathrm{~mm}$ diameter, $106 \mu \mathrm{Ci})$ was in the mid-plane and the rod source $(2.5 \mathrm{~mm}$ diameter, $118 \mu \mathrm{Ci})$ was in a plane that was $2 \mathrm{~cm}$ away.

(a)

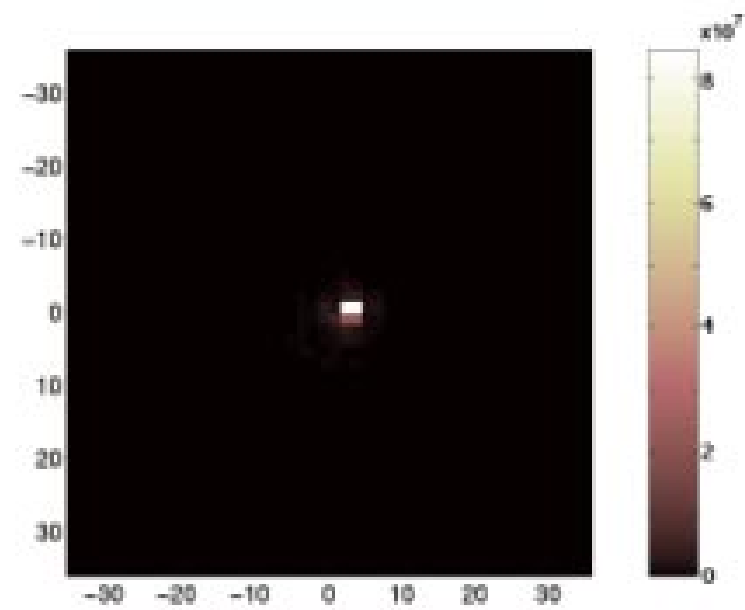

(b)

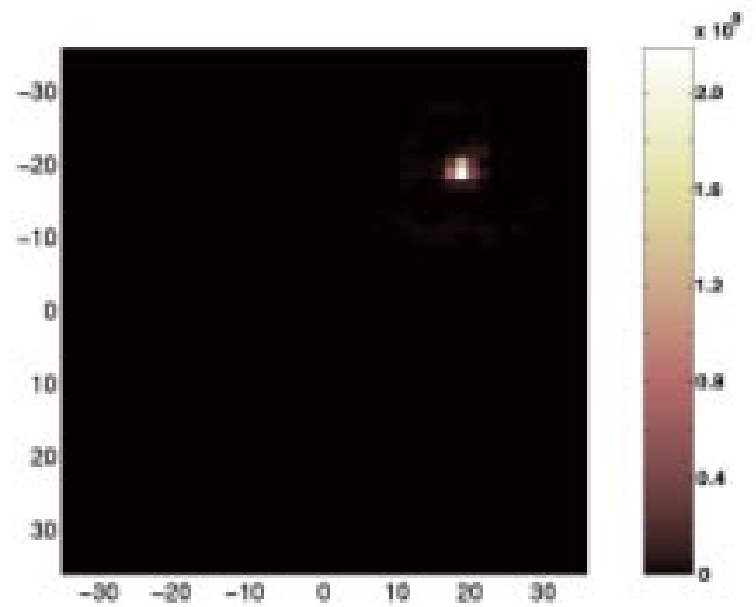

Fig. 9. Images of a $1.5 \mathrm{~mm}$ diameter ${ }^{68} \mathrm{Ge}$ point source $(106 \mu \mathrm{Ci})$ placed in the mid-plane, as described in Fig. 8a. The source position was not centered on a crystal. Data was taken at two different positions. The images were reconstructed with a maximum likelihood algorithm using $10 \mathrm{M}$ events. The $x$ - and $y$ - scale are in millimeters. (a) The reconstructed point in position 1 (near the center) has a $(1.65,2.85) \mathrm{mm}$ FWHM reconstruction resolution. The vertical intensity scale ranges from $0-8 \times 10^{7}$. (b) In position 2 (near the upper right corner), the reconstruction resolution is $(2.40,1.95)$ $\mathrm{mm}$ FWHM. The vertical intensity scale ranges from $0-2 \times 10^{8}$. 
(a)

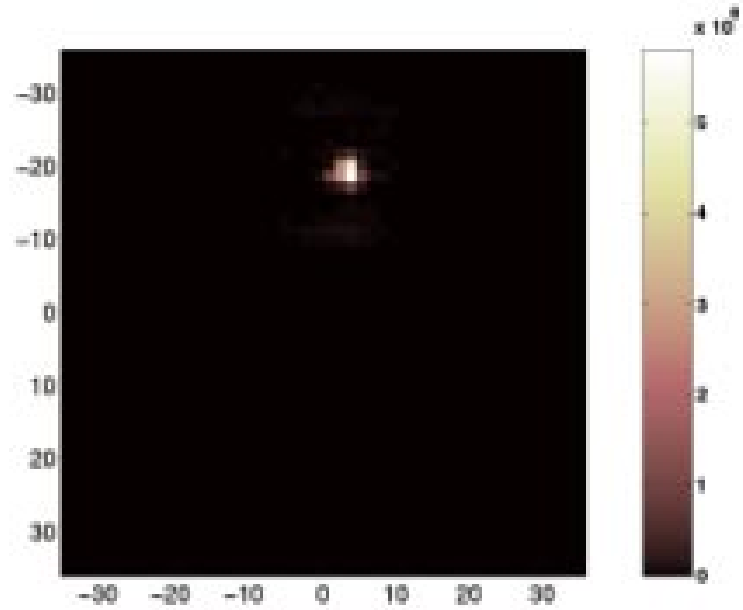

(b)

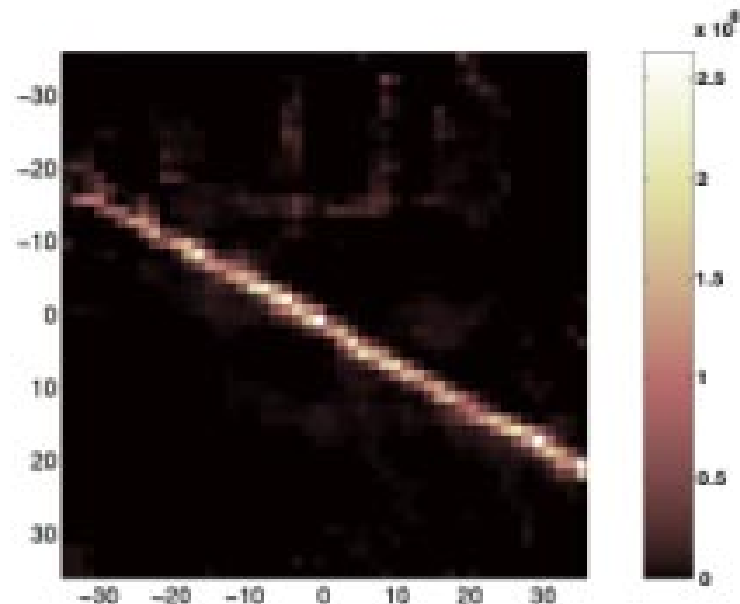

Fig. 10. Images of the (a) $1.5 \mathrm{~mm}$ diameter ${ }^{68} \mathrm{Ge}$ point source $(106 \mu \mathrm{Ci})$ placed in the mid-plane and (b) $2.5 \mathrm{~mm}$ diameter rod source $(118 \mu \mathrm{Ci})$ placed in a different plane ( $2 \mathrm{~cm}$ away), as described in Fig. $8 \mathrm{~b}$. The $\mathrm{x}-$ and $\mathrm{y}$ - scale are in millimeters. The vertical intensity scale ranges from $0-5 \times 10^{7}$ for (a) and from $0-2.5 \times 10^{6}$ for (b). The reconstruction was done using $10 \mathrm{M}$ events. The point source has $(3.00,2.25) \mathrm{mm}$ FWHM reconstruction resolution. Since the concentration of activity in the point source is much stronger than the rod source, background activity in the area of the point source is seen in the rod source plane.

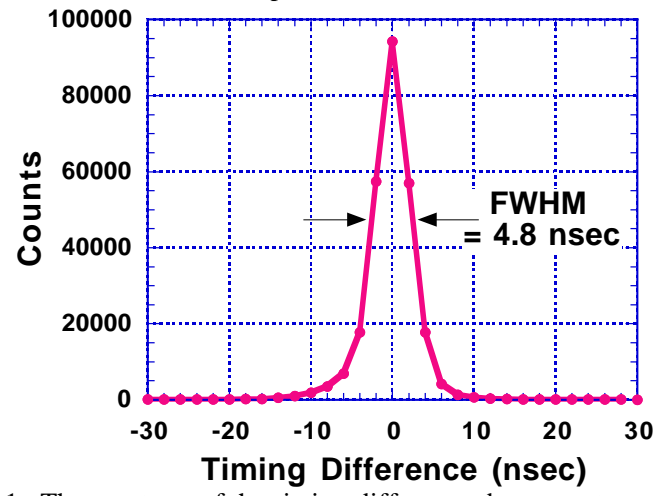

Fig. 11. The spectrum of the timing difference between two production detector modules, which were read out with the final data acquisition system. Data was taken using the $1.5 \mathrm{~mm}$ diameter ${ }^{68} \mathrm{Ge}$ point source (106 $\mu \mathrm{Ci})$ placed in the mid-plane between the two detector modules. Only the PMT information was used, with a low energy threshold. No channel to channel corrections were made. The preliminary coincidence timing resolution of $4.8 \mathrm{nsec}$ is expected to improve after timing calibration is complete. 